Pacific Journal of Mathematics

CALCULATIONS OF THE SCHUR GROUP 


\section{CALCULATIONS OF THE SCHUR GROUP}

\section{J. William Pendergrass}

Let the field $K$ be an abelian extension of the rational field $Q$. The Schur group of $K, S(K)$, consists of those classes in the Brauer group of $K$ which contain an algebra isomorphic to a simple component of a rational group algebra $Q G$ for some finite group $G$.

Suppose that $K$ has a cyclic extension of the form $Q(\zeta)$ where $\zeta$ is a primitive $n$th root of unity. In this paper we calculate the 2-part of $S(K)$ where $K$ contains the fourth roots of unity.

An interesting facet of these results is that in some cases certain local indices of classes in $S(K)$ are tied together. That is, a class in $S(K)$ must have a nontrivial local index at an even number of the primes in a certain set. The tying together of local indices in these fields is caused by quadratic reciprocity and is not found in the $q$-part of $S(K)$ where $q$ is an odd prime number.

Let $[A]$ be the class in the Brauer group of $K$ which contains the $K$-central simple algebra $A$. The Hasse invariant of $[A]$ at a prime $(S)$ of $K$ is denoted $\operatorname{inv}_{\mathbb{G}}[A]$. Benard and Schacher [2] showed that each class $[A]$ in $S(K)$ has uniformly distributed invariants. That is, if the index of $[A]$ is $I$, and $\sigma\left(\varepsilon_{I}\right)=\varepsilon_{I}^{\lambda}$ where $\varepsilon_{I}$ is a primitive $I$ th root of unity and $\sigma \in \operatorname{Gal}(K / Q)$, then $\operatorname{inv}_{\Theta}[A]=\lambda \operatorname{inv}_{\sigma(\Theta)}[A]$ for each prime (S) in $K$. A corollary of this result is that the local index of a class $[A]$ in $S(K)$ is the same at each of the primes of $K$ which divide a single rational prime $p$. This common index is called the $p$-local index of $[A]$.

Set $L=Q(\xi)$ where $\xi$ is a primitive $2^{s} n$th root of unity, $(2, n)=1$. Let $K$ be a field contained in $L$ such that $\operatorname{Gal}(L / K)=\langle\phi\rangle$ is a cyclic group of order $2^{t} t^{\prime},\left(2, t^{\prime}\right)=1$. Let $\zeta$ be a primitive $2^{s}$ th root of unity and suppose that $\phi(\zeta)=\zeta^{h}$ where $h=5^{2^{r-2}}$. Thus the $2^{r}$ th roots of unity lie in $K$. A theorem of Benard and Schacher [2] implies that the exponent of the 2-part of $S(K)$ is at most $2^{r}$.

Observe that there can be at most one rational prime $p$ with even ramification index in $L / K$. This follows from the fact that the inertia group of a divisor of $p$ is contained in Gal $(L / K(\varepsilon))$ where $\varepsilon$ is a root of unity in $L$ having largest possible order not divisible by $p$. If $p$ is such a prime, then let:

$2^{k}$ exactly divide $p-1$,

$2^{c}$ exactly divide $e(p, L / K)$,

$2^{d}$ exactly divide $f(p, K / Q)$, 
where $e(p, L / K)$ is the ramification index of $p$ in $L / K$ and $f(p, K / Q)$ is the residue class degree of $p$ in $K / Q$.

Now suppose that $q$ is a prime which does not divide $2 n$. We shall use the following notation:

$2^{l(q)}$ exactly divides $q-1$,

$2^{b(q)}$ exactly divides $f(q, K / Q)$,

$2^{a(q)}$ exactly divides $A(q)$ where $\phi^{A(q)}=[L / K, q]$ is the Frobenius automorphism of $q$ in $L / K$,

$2^{v(q)}$ exactly divides $V(q)$ where $h^{A(q)}-q^{f(q, K / Q)}=V(q) 2^{s}$. In addition, for any prime $p$ we denote $p^{f(p, K / Q)}-1$ by $\Gamma(p)$.

Finally let $\lambda=\max \{s-t, 0\}$.

THEOREM. The 2-part of $S(K)$ consists of those classes [A] in the Brauer group of $K$ which have uniformly distributed invariants which satisfy the following conditions.

(I) If $q$ does not divide $n$, then the $q$-invariants of [A] are integral multiples of $1 / 2^{I(q)}$ where

$$
I(q)=\left\{\begin{array}{l}
\max \{r-b(q), \iota(q)-v(q), 0\} \quad \text { if } \quad \iota(q) \leqq r-\lambda \\
\max \{r-b(q), r-\lambda-v(q), 0\} \quad \text { if } \quad \ell(q) \geqq r-\lambda .
\end{array}\right.
$$

(II) If $p$ divides $n$, then the $p$-invariants of [A] are integral multiples of $1 / 2^{I(p)}$ where

$$
I(p)=\left\{\begin{array}{l}
0 \text { if } p=2 \text { or if } e(p, L / K) \text { is odd } \\
\max \{c-d+r-k, c-d+s-t-\lambda, 0\} \text { otherwise. }
\end{array}\right.
$$

(III) Suppose that $p$ divides $n$ and $I(p) \neq 0$. If $\cdot k>s, k \neq t$, and $2^{k+s-t-\lambda}$ is greater than the power of 2 which divides $p^{\prime}-1$ for all primes $p^{\prime} \neq p$ which divide $n$, then the $q$-invariants of [A] are odd multiples of $1 / 2^{I(q)}$ for an even number of primes $q$ in the set.

$$
\{p\} \cup\{q:(q / p)=-1,(q, 2 n)=1 \text {, and } \measuredangle(q) \geqq r-\lambda\}
$$

where $(q / p)$ is the Legendre symbol.

Proof. Let $K^{\prime} \supset K$ be the field such that $\left[L: K^{\prime}\right]=2^{t}$. Then Lemma 2 of [5] implies that the set of permissible invariants for -elements in the 2-part of $S(K)$ is exactly the set of permissible invariants for elements in the 2-part of $S\left(K^{\prime}\right)$. Thus we may assume that $[L: K]=2^{t}$ without any loss of generality.

Now we must determine the invariants of the crossed product algebras of the form

$$
\left[L\left(\varepsilon_{q}\right) / K, \alpha\right]=\sum L\left(\varepsilon_{q}\right) u_{\sigma}, \quad \sigma \in \operatorname{Gal}\left(L\left(\varepsilon_{q}\right) / K\right)
$$

where $\varepsilon_{q}$ is a primitive $q$ th root of unity, $q$ is an odd prime which 
does not divide $n$, and $\alpha$ is a factor set from $\operatorname{Gal}\left(L\left(\varepsilon_{q}\right) / K\right) \times$ $\operatorname{Gal}\left(L\left(\varepsilon_{q}\right) / K\right)$ into $\langle\zeta\rangle$. The multiplication in these algebras is given by

$$
\begin{aligned}
& u_{\sigma} u_{\tau}=\alpha(\sigma, \tau) u_{\sigma \tau}, \\
& u_{\sigma} w=\sigma(w) u_{\sigma},
\end{aligned}
$$

for $\sigma, \tau \in \operatorname{Gal}\left(L\left(\varepsilon_{q}\right) / K\right)$ and $w \in L\left(\varepsilon_{q}\right)$. We know from Theorem 1 of [5] that the classes in the Brauer group of $K$ which contain these classes generate the 2-part of $S(K)$.

Let $\Delta_{q}=\Delta_{q}(x, y, z)$ be the algebra $\left(L\left(\varepsilon_{q}\right) / K, \alpha\right)$ where the values of $\alpha$ are in $\langle\zeta\rangle$ and $q$ is an odd prime not dividing $n$. Set $\operatorname{Gal}\left(L\left(\varepsilon_{q}\right) / L\right)=$ $\langle\gamma\rangle$. The factor set $\alpha$ is determined by the integers $x, y$, and $z$ where

$$
\begin{aligned}
& u_{\gamma} u_{\phi}=\zeta^{x} u_{\phi} u_{r}, \\
& \left(u_{\gamma}\right)^{q-1}=\zeta^{y}, \\
& \left(u_{\phi}\right)^{2^{t}}=\zeta^{z} .
\end{aligned}
$$

We must have

$$
\begin{aligned}
& u_{\phi}\left(\zeta^{z}\right)=\phi\left(\zeta^{z}\right) u_{\phi}=\zeta^{z} u_{\phi} \\
& u_{r}\left(\zeta^{y}\right)=\gamma\left(\zeta^{y}\right) u_{\gamma}=\zeta^{y} u_{\gamma} \\
& \left(u_{\gamma} u_{\phi} u_{\gamma}^{-1}\right)^{2^{t}}=\left(\zeta^{x} u_{\phi}\right)^{t} \\
& \left(u_{\phi} u_{\gamma} u_{\phi}^{-1}\right)^{q-1}=\left(\zeta^{-x} u_{\gamma}\right)^{q-1} .
\end{aligned}
$$

Thus

(a) $2^{s-r}$ divides $z$,

(b) $2^{\lambda}$ divides $x$,

(c) $y(h-1)+x(q-1)=Y 2^{s}$ for some integer $Y$.

The Frobenius automorphism of $q$ in $L / K$ is $\phi^{A(q)}$. Thus

$$
\phi^{A(q)}(\zeta)=\zeta^{h^{A(q)}}=\zeta^{q^{f(q, K Q)}} .
$$

Hence

$$
h^{A(q)}-q^{f(q, K / Q)}=V 2^{s} \text { for some integer } V .
$$

Now applying Theorem 3 of [6] we get that the $q$-local index of $\left[\Delta_{q}\right]$ is given by

$$
\frac{q-1}{(\nu(q)(q-1), q-1)}
$$

where

$$
\text { (a) } \begin{aligned}
\nu(q) & =\frac{1}{2^{s}}\left[x \frac{h^{A(q)}-1}{h-1}+y \frac{\Gamma(q)}{q-1}\right] \\
\text { (b) } \quad & =\frac{1}{h-1}\left[Y \frac{\Gamma(q)}{q-1}-x V\right] .
\end{aligned}
$$


Thus, since $2^{r}$ exactly divides $h-1$, the $q$-local index of $\left[\Delta_{q}\right]$ is $\max \left\{2^{r-\mu}, 1\right\}$ where $2^{\mu}$ exactly divides $Y \Gamma(q) /(q-1)-x V$.

We know that $2^{b(q)}$ exactly divides $\Gamma(q) /(q-1)$. Moreover, we may make $Y$ either odd or even without changing the power of 2 which divides $x$. If $\ell(q) \leqq r-\lambda$, then equation (1)(c) implies that $2^{r-l(q)}$ is the smallest power of 2 which can divide $x$. If $\ell \geqq r-\lambda$, then $2^{\lambda}$ is the least power of 2 which can divide $x$. Thus, the maximum $q$-local index of $\left[\Delta^{q}\right]$ is $2^{I(q)}$ where

$$
I(q)= \begin{cases}\max \{r-b(q), \iota(q)-v(q), 0\} & \text { if } \quad \ell(q) \leqq r-\lambda \\ \max \{r-b(q), r-\lambda-v(q), 0\} & \text { if } \quad \ell(q) \geqq r-\lambda .\end{cases}
$$

Now observe that for any prime $q^{\prime}$ which does not divide $2 n q$, $q$ is unramified in $L\left(\varepsilon_{q^{\prime}}\right) / K$. Thus the $q$-invariants of $\left[\Delta_{q^{\prime}}\right]$ must be zero. This means that the only classes amongst the generators of the 2-part of $S(K)$ which have non-zero invariants at the primes of $K$ dividing $q$ are those classes of the form $\left[\Delta_{q}(x, y, z)\right]$. Thus we have proved (I).

If there is no prime which ramifies in $L / K$, then $\left[\Delta_{q}\right]$ can have nonzero invariants only at the primes of $K$ which divide $q$. If 2 ramifies in $L / K$, it must be the only prime which ramifies in $L / K$. So, since the 2-invariants of any class in $S(K)$ must be zero by the results of Yamada [7], the only nonzero invariants that $\left[\Delta_{q}\right]$ can have are at the primes of $K$ which divide $q$. In both of these cases we are done and the theorem is proved.

So for the remainder of the proof let $p$ be an odd prime which is ramified in $L / K$. Set $\phi^{g^{\prime} \gamma^{g}}$ equal to a Frobenius automorphism for $p$ in $L\left(\varepsilon_{q}\right) / K$. Observe that ${\phi^{2 t-c}}^{2}$ generates the inertia group of $p$ in $L / K$ where $2^{c}=e(p, L / K)$.

Applying Theorem 3 of [6] we get that the $p$-local index of $\left[\Delta_{q}\right]$ is given by

$$
\frac{2^{c}}{\left(2^{c} \nu(p), 2^{c}\right)}
$$

where

$$
\nu(p)=\frac{1}{2^{s}}\left[-x g \frac{h^{2^{t-c}}-1}{h-1}+z \frac{\Gamma(p)}{2^{c}}\right] .
$$

Thus the $p$-local index of $\left[\Delta_{q}\right]$ is $\max \left\{2^{s-\eta}, 1\right\}$ where $2^{\eta}$ exactly divides

$$
-x g \frac{h^{2^{t-c}}-1}{h-1}+z \frac{\Gamma(p)}{2^{c}} \text {. }
$$

We know that $2^{t-c}$ exactly divides $\left(h^{2^{t-c}}-1\right) /(h-1)$, that $2^{k+d-c}$ exactly divides $\Gamma(p) / 2^{c}$, and that $2^{s-r}$ is the least power of 2 which divides $z$. Hence we need to find the smallest power of 2 which 
divides $x g$.

We know that $g$ must be an $f(p, K / Q)$ th power, so picking $q$ such that $(q / p)=-1$ we get that $\min \left\{2^{d}, 2^{\ell(q)}\right\}$ is the smallest power of 2 which can divide $g$. If $\zeta(q) \geqq r-\lambda$, then $2^{\lambda}$ must divide $x$, and if $\ell(q) \leqq r-\lambda$, then $2^{r-\ell(q)}$ must divide $x$. Hence we find that $\min \left\{2^{\lambda+d}, 2^{r}\right\}$ is the smallest power of 2 which can exactly divide $x g$. Thus the maximum $p$-local index of a class in the 2-part of $S(K)$ is $2^{I(p)}$ where

$$
\begin{aligned}
I(p) & =\max \{c-d+s-t-\lambda, c-t+s-r, c-d+r-k, 0\} \\
& =\max \{c-d+s-t-\lambda, c-d+r-k, 0\}
\end{aligned}
$$

since $c \leqq t-(s-r)$. This proves (II).

If $I(p)=0$, then we are finished. So assume for the rest of the proof that $I(p)>0$.

Now assume that $k>s, k \neq t$, and $2^{k+s-t-\lambda}$ is greater than the power of 2 which divides $p^{\prime}-1$ for all primes $p^{\prime}$ which are unequal to $p$ and which divide $n$.

Suppose that the $p$-local index of $\left[\Delta_{q}(x, y, z)\right]$ is $2^{I(p)}$. Now $s-$ $t-\lambda>r-k$ so $I(p)=c-d+s-t-\lambda$. Thus $2^{\lambda+d}$ exactly divides $x g$, indeed $2^{\lambda}$ must exactly divide $x$ and $2^{d}$ must exactly divide $g$. Thus $\ell(q) \geqq r-\lambda$ and $(q / p)=-1$. Further, since $p \equiv 1 \bmod 4$, $(p / q)=-1$ by the law of quadratic reciprocity. This, together with the hypotheses, implies that $b(q)=k-t+a(q)$ where $2^{b(q)}$ exactly divides $f(q, K / Q)$ and $a(q)$ exactly divides $A(q)$. Hence $\ell(q)+b(q)>$ $r+a(q)$. So, since $2^{\ell(q)+b(q)}$ exactly divides $q^{f(q)}-1$ and $2^{r+a(q)}$ exactly divides $h^{A(q)}-1$, we get that $r+a(q)=s+v(q)$. Thus

$$
r-b(q)=r-k+t-a(q)<r-\lambda-a(q) \leqq r-\lambda-v(q) .
$$

Hence $I(q)=r-\lambda-v(q)$ and the $q$-local index of $\left[\Delta_{q}(x, y, z)\right]$ is $2^{I(q)}$. Observe that $I(q)>0$ since the hypotheses insure that $a(q)<s-\lambda$, so that $v(q)<r-\lambda$.

Now let $q$ be a prime such that $(q / p)=-1,(q, 2 n)=1$, and $\measuredangle(q) \geqq r-\lambda$. Suppose that the $q$-local index of $\left[\Delta_{q}(x, y, z)\right]$ is $2^{I(q)}$. We have seen that $I(q)$ is positive and is equal to $r-\lambda-v(q)$ in this instance. Hence $2^{\lambda}$ must exactly divide $x$ by equation $(2)(b)$. Thus the $p$-local index of $\left[\Delta_{q}(x, y, z)\right]$ is greater than or equal to $2^{c-d+s-t-\lambda}$. However $I(p)=c-d+s-t-\lambda>0$, so the $p$-local index of $\left[\Delta_{q}(x, y, z)\right]$ must be $2^{I(p)}$.

We have now shown that under the hypotheses of (III), the $p$ local index of $\left[\Delta_{q}\right]$ is $2^{I(p)}$ if and only if $(q / p)=-1, \ell(q) \geqq r-\lambda$, and the $q$-local index of $\left[\Delta_{q}\right]$ is $2^{I(q)}$. This proves (III).

We now need to show that the restrictions on the invariants of elements in the 2-part of $S(K)$ given in the theorem are the only 
restrictions on the invariants of elements in the 2-part of $S(K)$.

First assume that the hypotheses of (III) hold. Let $F=$ $Q\left(\varepsilon_{p}, \varepsilon_{2^{s+1}}, \sqrt[4]{p}\right)$ and let $\sigma$ be the element in Gal $(F / Q)$ such that $\sigma\left(\varepsilon_{p}\right)=\varepsilon_{p}^{-1}, \quad \sigma(\sqrt[4]{p})=-\sqrt[4]{p}$, and $\sigma\left(\varepsilon_{2^{s+1}}\right)=\left(\varepsilon_{2^{s+1}}\right)^{\beta}$ where $\beta=5^{2^{s-2}}$. Such a $\sigma$ exists since $p$ does not have a fourth root in $Q\left(\varepsilon_{p}, \varepsilon_{2^{8+1}}\right)$. Let $q$ be a prime not dividing $n$ whose Frobenius automorphism in $F / K$ is $\sigma$. There are infinitely many such primes by the Tchebotarev density theorem. This means that $2^{s}$ exactly divides $q-1,2$ exactly divides $f\left(q, Q\left(\varepsilon_{p}\right) / Q\right)$, and $2^{s-1}$ exactly divides $f\left(p, Q\left(\varepsilon_{q}\right) / Q\right)$. Thus the Frobenius automorphism of $q$ in $L / K$ is an odd power of $\phi^{2^{t-1}}$ if $f(q, K / Q)$ is odd, and it is 1 if $f(q, K / Q)$ is even. So we have that $a(q)=t-1$ if $f(q, K / Q)$ is odd, and $a(q)=0$ if $f(q, K / Q)$ is even. Further $\ell(q) \geqq r-\lambda$.

Now the algebra class $\left[\Delta_{q}\left(2^{\lambda}, 0,0\right)\right]$ has $q$-local index 1 if $f(q, K / Q)$ is even and $\left[\Delta_{q}\left(2^{\lambda}, 2^{s-1}, 0\right)\right]$ has $q$-local index 1 if $f(q, K / Q)$ is odd. This follows from equation (2)(a). Now both of these algebra classes have $p$-local index $2^{I(p)-1}$ since $2^{\lambda+d+1}$ divides $x g$ in both cases. Thus the algebra class which has local index $2^{I(p)-1}$ at $p$ and local index 1 at all other primes is in $S(K)$. This implies that there are no further restrictions on the 2-part of $S(K)$ in the case where the hypotheses of (III) hold.

Now assume that either $k \leqq s$ or $k=t>s$. Let $\psi_{p}$ be a generator of $\operatorname{Gal}\left(L / Q\left(\zeta, \varepsilon_{n^{*}}\right)\right)$ where $\left(n^{*}, p\right)=1$ and $n / n^{*}$ is a power of $p$. Also set $\psi$ equal to the automorphism in $\operatorname{Gal}\left(L / Q\left(\varepsilon_{n}\right)\right)$ which sends $\zeta$ to $\zeta^{5}$. Now let $q^{\prime}$ be a prime whose Frobenius automorphism in $L / Q$ is $\psi_{p} \psi^{2^{r+t-k-2}}$. This implies that $\left(q^{\prime} / p\right)=\left(p / q^{\prime}\right)=-1$, that $2^{r+t-k}$ exactly divides $q^{\prime}-1$, and that $2^{a+k-t}$ exactly divides $f\left(q^{\prime}, K / Q\right)$. Consider the algebra class $\left[\Delta_{q},\left(x_{0}, y_{0}, 0\right]\right.$ where

$$
x_{0} \equiv 2^{\lambda-b\left(q^{\prime}\right)}\left[\frac{\Gamma\left(q^{\prime}\right)}{q^{\prime}-1}\right] \bmod 2^{s}
$$

and

$$
y_{0} \equiv-2^{\lambda-b\left(q^{\prime}\right)}\left[\frac{h^{A\left(q^{\prime}\right)}-1}{h-1}\right] \bmod 2^{s} .
$$

Observe that $x_{0}\left(q^{\prime}-1\right)+y_{0}(h-1) \equiv 0 \bmod 2^{s}$ so that equation (1)(c) is satisfied. Now we have that

$$
x_{0}\left[\frac{h^{A\left(q^{\prime}\right)}-1}{h-1}\right]+y_{0}\left[\frac{\Gamma\left(q^{\prime}\right)}{q^{\prime}-1}\right] \equiv 0 \bmod 2^{s} .
$$

Hence the $q^{\prime}$-local index of $\left[\Delta_{q^{\prime}}\left(x_{0}, y_{0}, 0\right)\right]$ is 1 . Further, $2^{\lambda}$ exactly divides $x_{0}, 2^{r-\lambda}$ divides $q^{\prime}-1$, and $\left(q^{\prime} / p\right)=-1$. Thus the $p$-local index of $\left[\Delta_{q^{\prime}}\left(x_{0}, y_{0}, 0\right)\right]$ is $\max \left\{2^{c-d+8-t-\lambda}, 1\right\}$.

Now consider the algebra class $\left[\Delta_{q^{\prime}}\left(0,0,2^{s-r}\right)\right]$. Its $q^{\prime}$-local index 
is 1 and its $p$-local index is $\max \left\{2^{c-d+r-k}, 1\right\}$.

Thus $S(K)$ contains the algebra class with local index $2^{I(p)}$ at $p$ and local index 1 at all other primes. This implies that there are no extra restrictions on the 2-part of $S(K)$ when either $k \leqq s$ or $k=t$.

Finally assume that $k>s, k \neq t$, and that there is a prime $p^{\prime} \neq p$ which divides $n$ such that $2^{k+s-t-\lambda}$ divides $p^{\prime}-1$. Let $\psi_{p^{\prime}}$ be a generator of $\operatorname{Gal}\left(L / Q\left(\zeta, \varepsilon_{n^{\prime}}\right)\right)$ where $\left(n^{\prime}, p^{\prime}\right)=1$ and $n / n^{\prime}$ is a power of $p^{\prime}$. Let $\psi_{p}$ be as above.

Let $q^{\prime \prime}$ be a prime whose Frobenius automorphism in $L / Q$ is $\psi_{p} \psi_{p^{\prime}}$. Thus $\left(q^{\prime \prime} / p\right)=-1$ and $2^{s}$ divides $q^{\prime \prime}-1$. Further observe that if $\beta$ is the smallest integer such that $\left(\psi_{p} \psi_{p^{\prime}}\right)^{\beta} \in \operatorname{Gal}(L / K)$, then $2^{k+s-t-\lambda}$ must divide $\beta$. Hence $a\left(q^{\prime \prime}\right) \geqq s-\lambda$. Thus $\left[\Delta_{q^{\prime \prime}}\left(2^{\lambda}, 0,0\right)\right]$ has $q^{\prime \prime}$-local index 1 and $p$-local index $2^{c-d+s-t-\lambda}$. Since $k>s \geqq r$, we have that $I(p)=c-d+s-t-\lambda$. So $S(K)$ contains an algebra with local index $2^{I(p)}$ at $p$ and local index 1 at all other primes. This implies that there are no further restrictions on the 2-part of $S(K)$ in this case.

This completes the proof to the theorem.

\section{REFERENCES}

1. M. Benard, The Schur subgroup I, J. of Algebra, 22 No. 2 (1972), 374-377.

2. M. Benard and M. Schacher, The Schur subgroup II, J. of Algebra, 22, No. 2 (1972), 378-385.

3. G. J. Januz, Generators for the Schur group of local and global number fields, Pacific J. Math., (to appear).

4. - The Schur group of an algebraic number field, University of Illinois preprint, Urbana Ill. 1975.

5. J. W. Pendergrass, The Schur subgroup of the Brauer group.

6. Y. Yamada, Characterization of the simple components of the group algebras over the p-adic number field, J. Math. Soc. Japan, 23 (1971) 295-310.

7. — Cyclotomic algebras over a 2-adic field, Proc. Japan Acad., 49, No. 6 (1973) 438-442.

8. - The Schur subgroup of the Brauer group, I, J. of Algebra, 27, No. 3 (1973) 579-589.

Received February 14, 1975

New Mexico State University 



\section{PACIFIC JOURNAL OF MATHEMATICS}

EDITORS

RICHARD ARENS (Managing Editor)

University of California

Los Angeles, California 90024

R. A. Beaumont

University of Washington

Seattle, Washington 98105
J. DUGUNDJI

Department of Mathematics

University of Southern Californı

Los Angeles, California 90007

D. Gilbarg and J. Milgram

Stanford University

Stanford, California 94305

\section{ASSOCIATE EDITORS}

E. F. BECKENBACH

B. H. NeumanN

F. WOLF

K. YoSHIDA

\section{SUPPORTING INSTITUTIONS}

UNIVERSITY OF BRITISH COLUMBIA

CALIFORNIA INSTITUTE OF TECHNOLOGY

UNIVERSITY OF CALIFORNIA

MONTANA STATE UNIVERSITY

UNIVERSITY OF NEVADA

NEW MEXICO STATE UNIVERSITY

OREGON STATE UNIVERSITY

UNIVERSITY OF OREGON

OSAKA UNIVERSITY
UNIVERSITY OF SOUTHERN CALIFORNIA

STANFORD UNIVERSITY

UNIVERSITY OF TOKYO

UNIVERSITY OF UTAH

WASHINGTON STATE UNIVERSITY

UNIVERSITY OF WASHINGTON

AMERICAN MATHEMATICAL SOCIETY

NAVAL WEAPONS CENTER 


\section{Pacific Journal of Mathematics}

Vol. 69 , No. 1

May, 1977

V. V. Anh and P. D. Tuan, On starlikeness and convexity of certain analytic

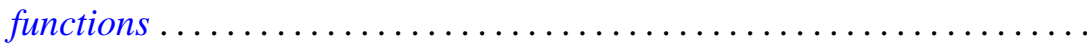

Willard Ellis Baxter and L. A. Casciotti, Rings with involution and the prime

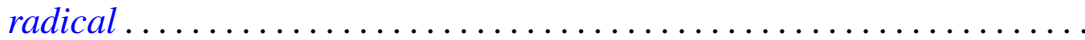

Manuel Phillip Berriozabal, Hon-Fei Lai and Dix Hayes Pettey,

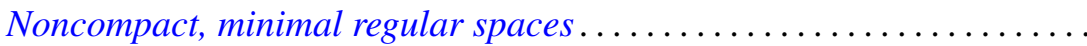

Sun Man Chang, Measures with continuous image law ................

John Benjamin Friedlander, Certain hypotheses concerning

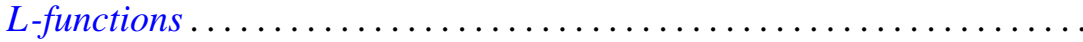

Moshe Goldberg and Ernst Gabor Straus, On characterizations and

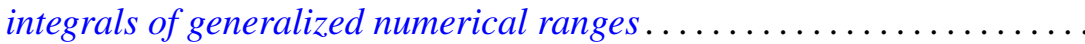

Pierre A. Grillet, On subdirectly irreducible commutative semigroups ...... 55

Robert E. Hartwig and Jiang Luh, On finite regular rings ..............

Roger Hugh Hunter, Fred Richman and Elbert A. Walker, Finite direct sums of cyclic valuated p-groups ........................... 97

Atsushi Inoue, On a class of unbounded operator algebras. III ......... 105

Wells Johnson and Kevin J. Mitchell, Symmetries for sums of the Legendre

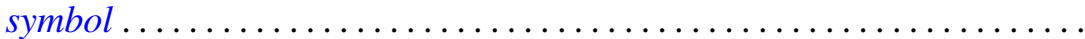

Jimmie Don Lawson, John Robie Liukkonen and Michael William Mislove,

Measure algebras of semilattices with finite breadth

Glenn Richard Luecke, A note on spectral continuity and on spectral properties of essentially $G_{1}$ operators ...............

Takahiko Nakazi, Invariant subspaces of weak-* Dirichlet algebras . .

James William Pendergrass, Calculations of the Schur group ...

Carl Pomerance, On composite $n$ for which $\varphi(n) \mid n-1$. II. . .

Marc Aristide Rieffel and Alfons Van Daele, A bounded operator approach to Tomita-Takesaki theory........................

Daniel Byron Shapiro, Spaces of similarities. IV. $(s, t)$-families ...

Leon M. Simon, Equations of mean curvature type in 2 independent variables.

Joseph Nicholas Simone, Metric components of continuous images of ordered compacta ............................

William Charles Waterhouse, Pairs of symmetric bilinear forms in

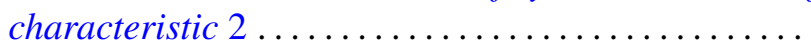

